\title{
Laser micromachining of optical microstructures with inclined sidewall profile
}

\author{
X. H. Wang, P. T. Lai, and H. W. Choi ${ }^{a}$ \\ Department of Electrical and Electronic Engineering, Semiconductor Lighting and Display Laboratory, \\ The University of Hong Kong, Pokfulam Road, Hong Kong
}

(Received 29 December 2008; accepted 16 March 2009; published 20 April 2009)

\begin{abstract}
Laser micromachining has been proved to be a useful tool for the formation of microstructures in semiconductor and optical materials. It is also widely adopted for dicing of light-emitting diode chips. The authors propose a modified laser micromachining setup which enables three-dimensional structures to be formed. A mirror is inserted in the optical path between the focusing optics and the machining plane so that the beam strikes the sample at an oblique angle. By translating and/or rotating the sample as micromachining is carried out, various three-dimensional structures such as a pyramid or a conic section can be obtained. Trenches as small as $10 \mu \mathrm{m}$ on sapphire have been realized with nanosecond ultraviolet laser pulses. Laser-induced damage, due to resolidification of the ablation melt, accumulates with increasing scans of the beam; it can be removed by chemical and mechanical treatment. (C) 2009 American Vacuum Society. [DOI: 10.1116/1.3117344]
\end{abstract}

\section{INTRODUCTION}

Micromachining with nanosecond laser pulses is a powerful tool that is suitable for replacing or complementing traditional wafer processes such as dicing and etching, as well as advanced process developments such as laser lift-off, ${ }^{1}$ laser-assisted machining, ${ }^{2}$ and rapid prototyping. ${ }^{3}$ Tightly focused nanosecond laser pulses enable micromachining with much higher precision with dimensions down to several micrometers. ${ }^{4-6}$ For more advanced applications, considerations such as laser wavelength, pulse energy, repetition rate, and pulse duration should be seriously taken into account. Drilling and cutting with nanosecond or even femtosecond ultraviolet (UV) laser pulses have been reported to excel with very small heat-affected zones. ${ }^{5-7}$ Laser micromachining is gradually being adopted for gallium nitride $(\mathrm{GaN})$ based light-emitting diodes (LEDs). Since the epitaxial GaN layers are typically grown on sapphire, separation of fabricated LED dies is commonly achieved by wafer sawing, which is slow and expensive. Using high energy laser pulses not only increases the process efficiency but also enables a high packing density of chips by virtue of the reduced dimensions of the scribe lanes.

One of the typical configurations of laser micromachining relies on a laser scanner head which steers the beam into the incident direction; ${ }^{5}$ although it is relatively fast, mechanical vibrations tend to be magnified, resulting in loss of pattern resolution. Alternatively, the sample to be micromachined can be mounted onto a precision motorized translation module while the optical beam remains static; this is more suitable for the processing of optical microstructures. In a conventional laser micromachining setup for wafer dicing, the focused laser beam is incident perpendicularly onto the sample to be processed, so that only two-dimensional patterns can be generated and vertical cuts can be obtained.

a) Author to whom correspondence should be addressed; electronic mail: hwchoi@hku.hk
Projecting the beam at an oblique angle to the sample enables three-dimensional micromachining. Nevertheless, it is not viable to mount the translation module at a tilted angle, as it will result in severe beam distortion. In our proposed approach, a laser beam turning mirror was introduced into the optical path to achieve a continuously tunable range of tilting angle for beam projection while retaining beam quality.

\section{EXPERIMENTAL DETAILS}

The setup for laser micromachining consists of an UV laser source, beam focusing optics, and an $x-y$ motorized translation stage. The laser source is a third harmonic neodymium-doped yttrium lithium fluoride diode-pumped solid state laser manufactured by Spectra Physics. The laser emits at $349 \mathrm{~nm}$, while the pulse repetition rate ranges from single pulse to $5 \mathrm{kHz}$. At a reference diode current of $3.2 \mathrm{~A}$, the pulse energy is $120 \mu \mathrm{J}$ at a repetition rate of $1 \mathrm{kHz}$, with a pulse width of around 4 ns. The TEM00 beam allows for tight focusing, offering high spatial resolution. After beam expansion and collimation through a beam expander, the laser beam is reflected $90^{\circ}$ by a dielectric laser line mirror and focused onto the horizontal machining plane to a very tiny spot several micrometers in diameter with a focusing triplet. All optics used are made of UV fused silica and are antireflection coated. The additional feature of our setup, as illustrated in the schematic of Fig. 1, is the insertion of an UV mirror at an oblique angle within the optical path between the focusing optics and the machining plane, which serves to deflect the convergent beam to strike the sample at an oblique angle with respect to the horizontal working plane. The size of the beam at the focal point not only is limited by the capability of the UV objective lens but is also sensitive to the coaxiality of the optics. With this modified setup, it is relatively easy to optimize and monitor the beam through the tube lens imaged with a charge coupled device camera. Once the optical setup is optimized before insertion of the tilting 


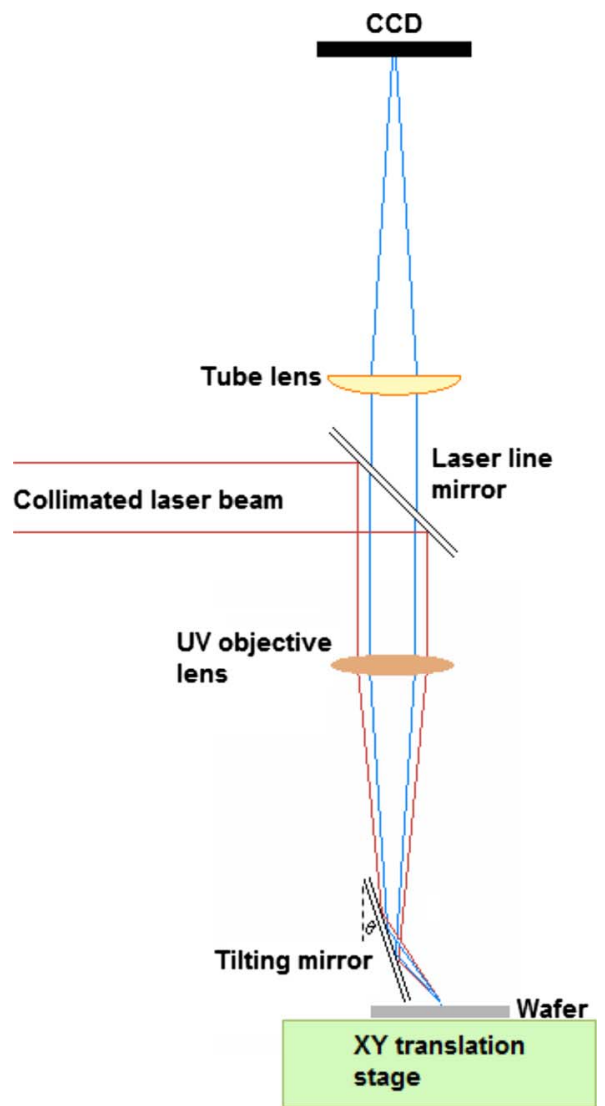

FIG. 1. (Color online) Schematic of laser micromachining setup.

mirror, the mirror can be inserted without affecting the coaxiality of the laser beam, so that the dimension of the beam spot remains unaffected.

The beam can be effectively applied for microsectioning with nonvertical sidewall profiles. The angle of incidence of the deflected laser beam on the wafer is $2 \theta$, where $\theta$ as indicated in Fig. 1 is the angle between the plane of the mirror to the normal. This angle is readily and precisely controlled by mounting the mirror onto a rotation stage; thus, the incident angle can be varied over a wide range. In this experiment, we have used an UV objective with a focal length of $75 \mathrm{~mm}$ based on two considerations. First, the focal length should be long enough to accommodate the mirror in the optical path. Second, an ideal tool for the fabrication of microstructures should have a very long penetration depth and negligible lateral dispersion. Nevertheless, an objective lens with a longer focal length also produces a larger focused beam spot. The two parameters are related via the following equation:

$$
d=\frac{4 \lambda M^{2} f}{\pi D}
$$

where $M^{2}$ quantifies the beam quality, $\lambda$ is the wavelength of the laser beam, $f$ is the focal length, and $D$ is the diameter of the incident beam.
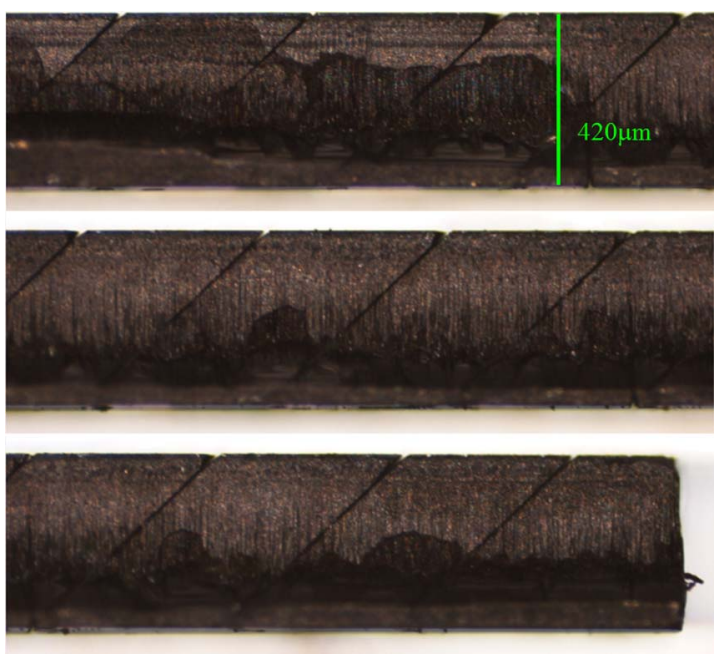

FIG. 2. (Color online) Cross-sectional optical microphotograph of laser micromachined microtrenches at an inclination of $\sim 45^{\circ}$ at a range of scan cycles of between 1 and 10 .

\section{RESULTS AND DISCUSSIONS}

As our scheme of micromachining is targeted at die separation of GaN LEDs, sapphire wafers are used for testing the results, as it is the typical substrate for the metal organic chemical vapor deposition growth of GaN. The quality of the cleave can be quantified by the width, depth, linearity, and sidewall roughness of the trench formed by the laser beam. Each of these parameters will be investigated. Since the focal length of the focusing lens $(f=75 \mathrm{~mm})$ is much longer than the thickness of the sapphire wafer $(t=420 \mu \mathrm{m})$, the depth of the trench mainly depends on the number of micromachining cycles. The number of cycles is controlled by configuring the translation stage to repeat its linear path over a number of times. Since the position repeatability of the stage is better than $5 \mu \mathrm{m}$, increasing the number of cycles should not contribute significantly to the width of the feature. Figure 2 shows the cross-sectional optical image of a $420 \mu \mathrm{m}$ thick sapphire wafer that has been micromachined with an incident beam inclined at $45^{\circ}$ or $60^{\circ}$, with scan cycles ranging from 1 to 10 . These incisions were carried out by setting the laser pulse energy to $54 \mu \mathrm{J}$ at a repetition rate of $2 \mathrm{kHz}$. The relationship between the inclined cutting depth and the number of passes of the beam is plotted in Fig. 3. After the first pass of the beam, a narrow trench with a width of $\sim 20 \mu \mathrm{m}$ and a depth of $\sim 220 \mu \mathrm{m}$ was formed. Successive scans of the beam along the trench results in further deepening and widening, but the extent was increasing less. The depth of the trench depends on the effective penetration of the beam. From the second scan onwards, the beam has to pass through the narrow gap before reaching the bottom of the trench for further machining. The energy available at this point is attenuated, partly due to lateral machining of the channel (causing undesirable widening), absorption, and diffraction effects. Therefore, the depth of the trench tends to saturate after multiple scans. For comparison, vertical trenches were micromachined with the tilting mirror removed, an example 


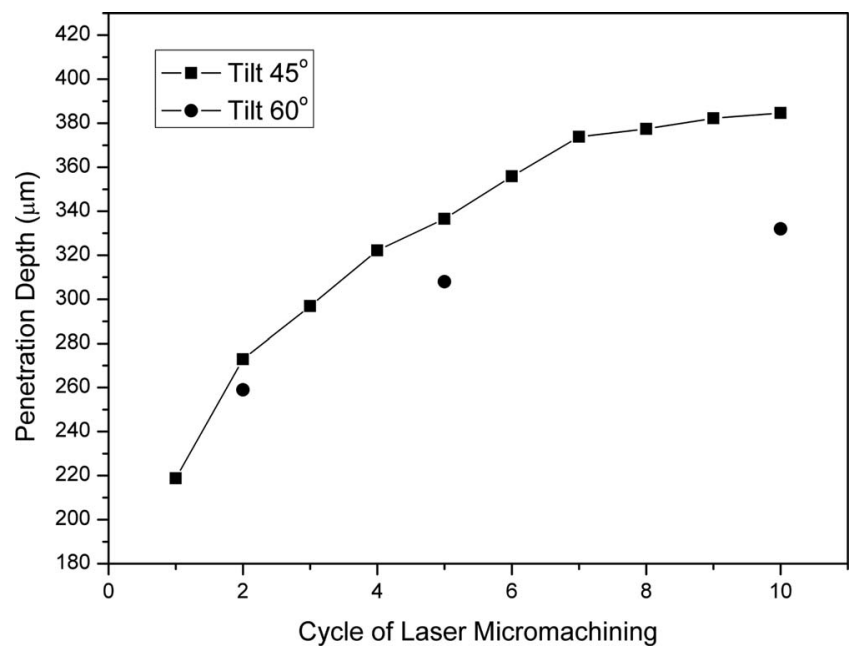

FIG. 3. Depth of microtrenches as a function of scan cycles for beam incident angles of $45^{\circ}$ and $60^{\circ}$.

of which is shown in the cross-sectional optical image of Fig. 4(a). Both the intersecting vertical and oblique cuts were formed after three passes of the scanning beam. A threedimensional (3D) field-emission scanning-electron microscope (FE-SEM) view of the same structure with the triangular section removed is shown in Fig. 4(b). It is worth noting that after the insertion of an additional tilting mirror, the ablation efficiency is reduced, the extent of which scales inversely with the angle of the incident beam, as is evident from the plot in Fig. 3. This may be attributed to polarization-dependent reflectivity and deformation of the beam spot.

Apart from linear micromachining, it is also possible to create three-dimensional microstructures using this modified micromachining scheme. One example is rotary micromachining for the preparation of conic structures. In this case, the sample is mounted on a motorized rotation stage while the beam is focused and incident at a fixed distance $R$ from the rotary axis. The sample is micromachined while the stage is rotated. Since the incident beam is tilted at an oblique angle, an inverted conical structure is drilled through the sapphire substrate after 10 cycles of rotation, as illustrated in Figs. 5(a) and 5(b). The radius of the top brim is determined by the position of the incident beam with respect to the rotary axis, while the radius of the lower brim depends on the incident angle and the depth of the conics (and thus the number of rotations of the beam). The top and bottom radii, as measured from the plan view optical microphotograph in Fig. 5(b), are $\sim 420$ and $\sim 140 \mu \mathrm{m}$, respectively. Since the incident angle of the beam was tuned to $45^{\circ}$, the length of the inclined surface is estimated at $400 \mu \mathrm{m}$. Deviation of circularity mainly stems from microscale wobble of the rotary stage.

The formation of inclined profiles with laser micromachining in semiconductor and optical materials can potentially be exploited to create chip geometries which can assist with light extraction, such as the truncated-inverted-pyramid structure $^{8}$ or the inverted conical structure as illustrated in

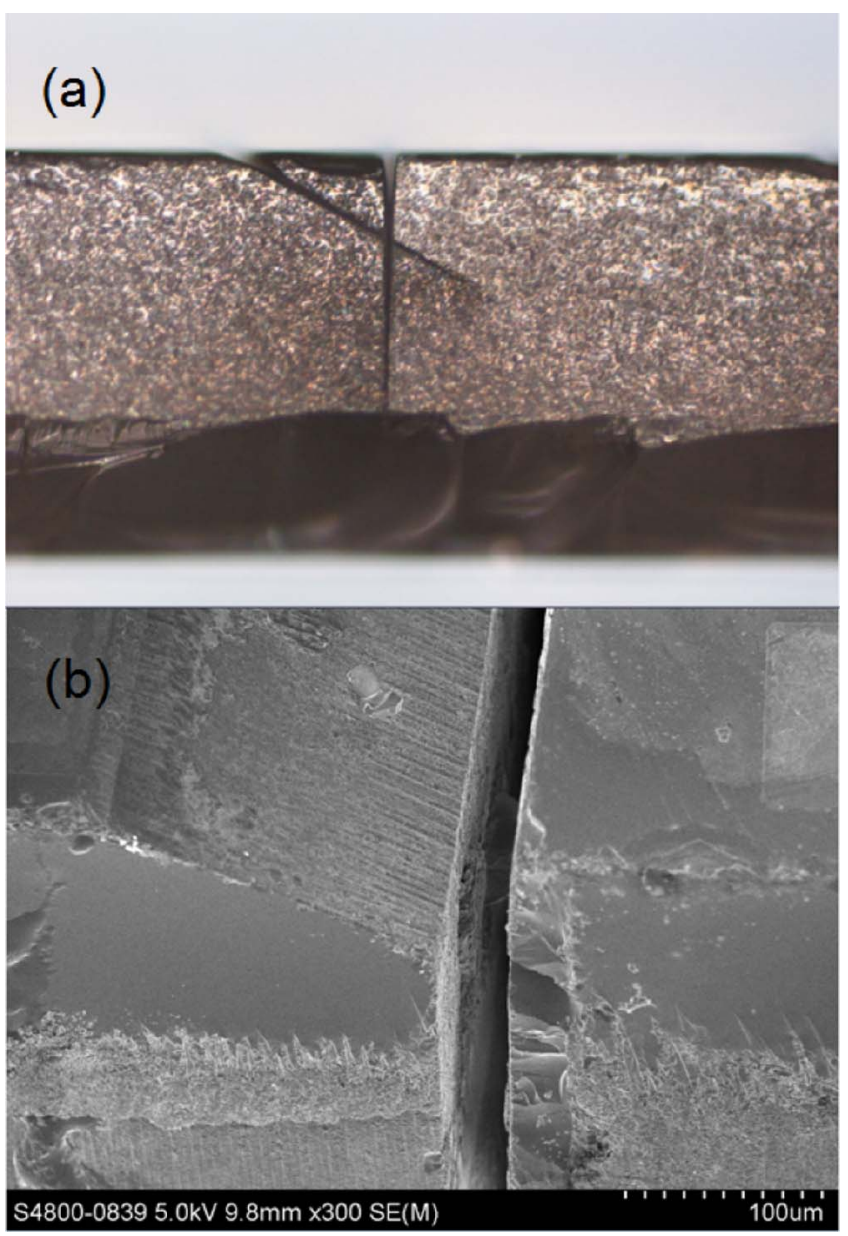

FIG. 4. (Color online) (a) Cross-sectional optical image showing an intersecting vertical ( 3 cycles) and an oblique cut $\left(\sim 60^{\circ}, 5\right.$ cycles) on sapphire. (b) FE-SEM image showing the 3D view of the structure in (a), with the triangular column removed.

Fig. 4. The inclined plane or curved surface can be mirror coated to act as a reflector in LEDs, enhancing light extraction efficiency and narrowing emission patterns. This technology can also be applied in the fabrication of other devices featuring diversified three-dimensional microstructures, such as devices and structures for optical communication, ${ }^{9,10}$ microfluidic chemical channel, ${ }^{11}$ and medical applications. ${ }^{12}$ The material used in our study - sapphire-is known to be the second hardest material; repeating the results in most other materials should be trivial. The sedimentary by-product from laser ablation in the form of metal oxide and nitride can be removed by soaking in diluted (18\%) $\mathrm{HCl}$ solution for 5 min, followed by sonication in de-ionized water. The optical microphotographs in Figs. 6(a) and 6(b) show the surface conditions of a laser-ablated sample before and after the cleaning process, testifying to the effectiveness of damage removal.

After the chemical treatment, the surface morphologies of the micromachined samples are examined with atomic force microscopy (AFM). 3D images of the AFM scans are shown in Fig. 7. The surface topography of the sapphire surface after two machining cycles exhibits a uniform roughness 

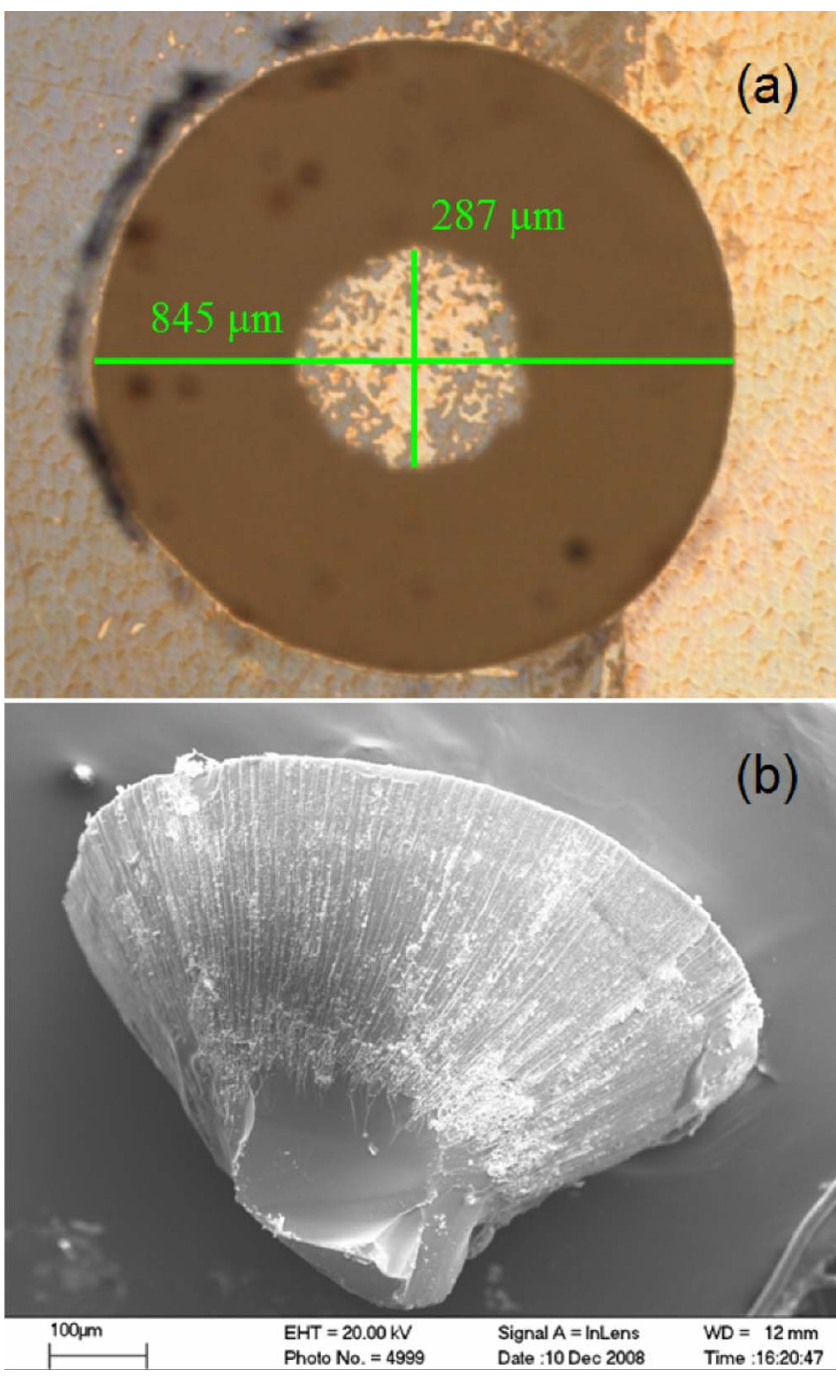

FIG. 5. (Color online) Laser micromachined conic section in (a) plan view and (b) $3 \mathrm{D}$ view.

with a rms. value of $\sim 150 \mathrm{~nm}$. With more cycles, increasing densities and dimensions of granules are observed on the AFM image and the rms roughness increased to $\sim 218 \mathrm{~nm}$ after 5 cycles. The formation of the larger grains on the surface is a result of uneven aggregation and resolidification of the melted material. The evacuation rate of the ablated species declines as the beam reaches deeper into the trench, and

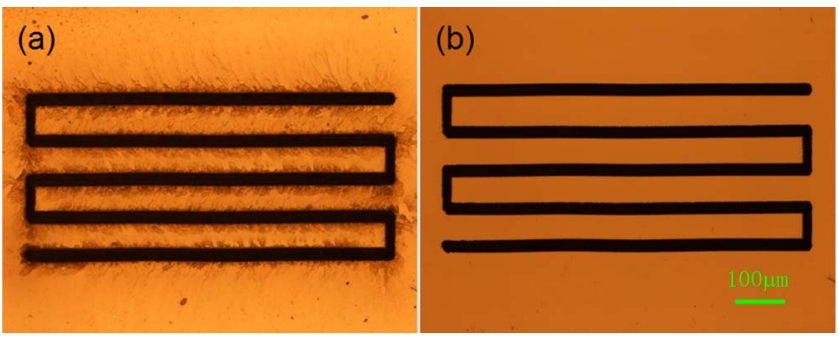

FIG. 6. (Color online) (a) Surface optical microphotograph of a laserpatterned sapphire sample prior to cleaning treatment. (b) The same sample after a cleaning process of soaking in $18 \% \mathrm{HCl}$ solution for $5 \mathrm{~min}$, followed with $20 \mathrm{~s}$ of sonication.

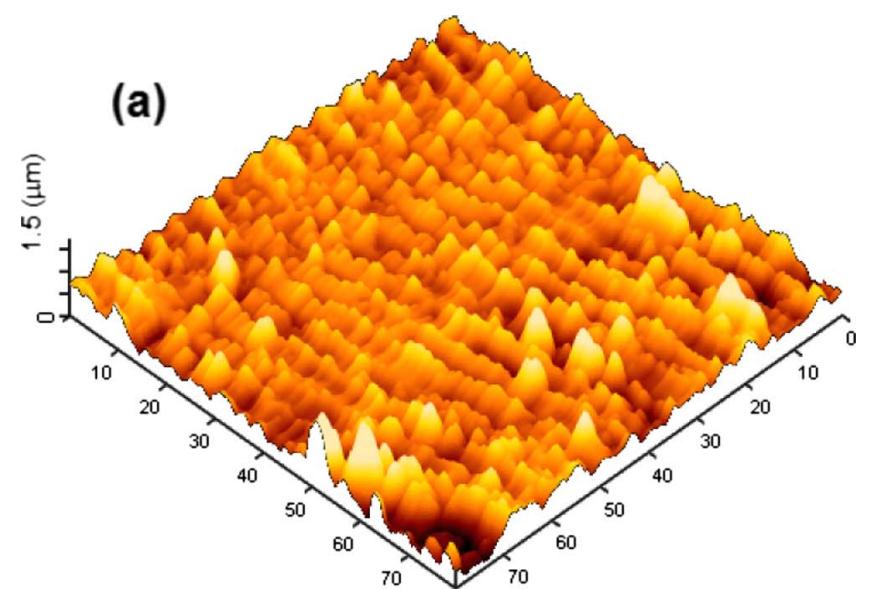

$[\mu \mathrm{m}]$

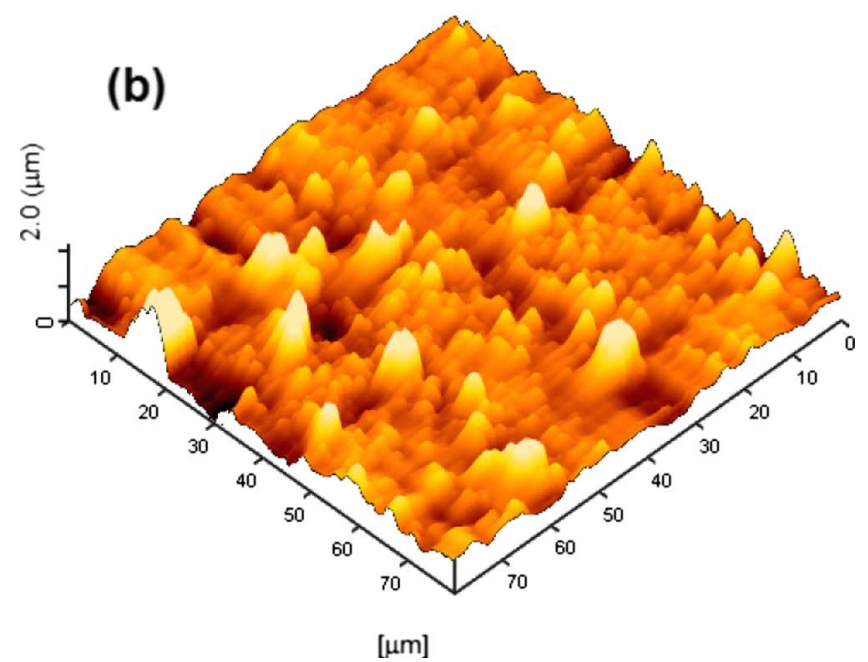

FIG. 7. (Color online) AFM morphology images of the inclined sapphire surfaces after laser micromachining for (a) 2 cycles and (b) 5 cycles. The corresponding rms roughnesses are 150 and $218 \mathrm{~nm}$, respectively.

statistically the density of aggregation is more pronounced at these deeper sites. After successive scan cycles, the aggregation of the melt during micromachining resulted in uneven sidewall roughening. An inclined surface machined with 10 cycles exhibits different rms roughnesses at different depths, making it difficult to find a representative value. This finding is corroborated with the FE-SEM images, as shown in Figs. $8(a)-8(f)$. The micromachined surface generally consists of a streaky patterning running along the inclined plane, which may be due to wobbling of the stage during linear motion. On top of this pattern are clusters of solids due to melt resolidification. The scale of these clusters increases in area upon increasing scan cycles. Nevertheless, these clusters are typically very thin and easily removed with a chemical and mechanical post-treatment. Laser micromachining is a nonlinear process that begins with the absorption of the laser pulse, followed by material breakdown and ablation. Generally, shorter pulse widths help minimize the heat-affected zone and produce sharp features. The nanosecond laser pulses used in our experiment proves its capability for this purpose. 

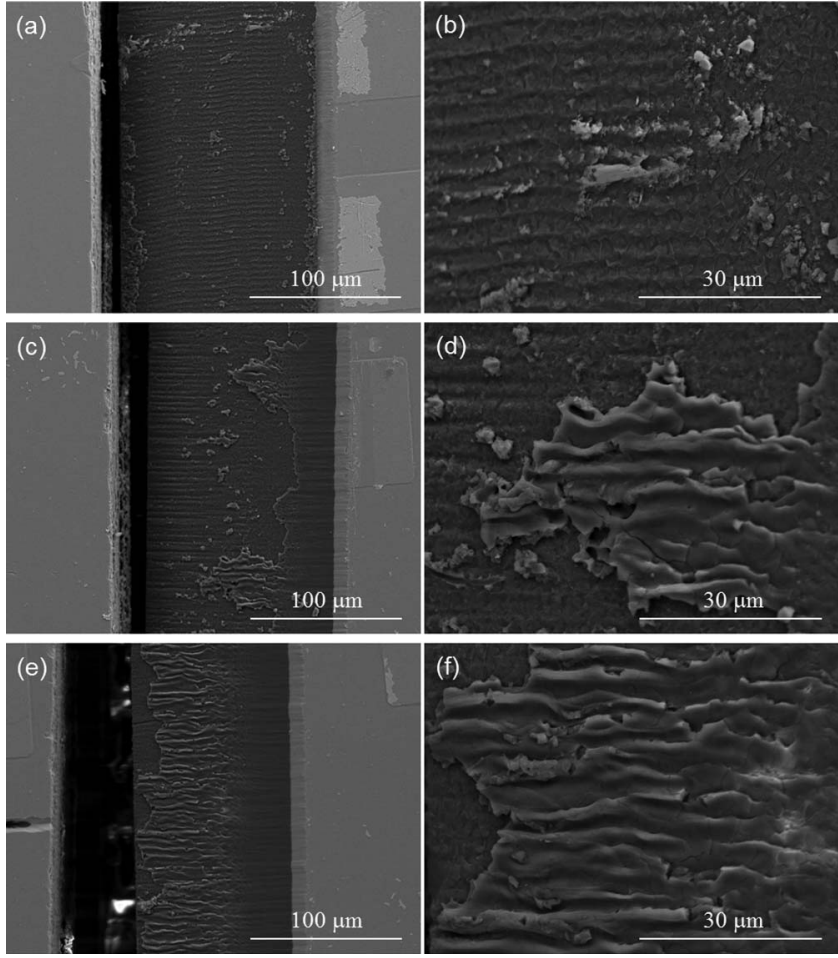

FIG. 8. Low and high magnification FE-SEM images of the inclined micromachined surfaces after $[(a)$ and (b) $] 2$ cycles, $[(c)$ and (d) 5 cycles. and $[(e)$ and (f)] 10 cycles.

As the pulse fluence from the laser far exceeds the critical fluence, ablation takes place in the very early stages of pulse deposition within subnanosecond or even shorter time scale, disregarding the specific mechanisms of the nonlinear optical process. The continuing plasma absorption of the remaining pulse and heating of the ions is accompanied by the expulsion of the ablated vapor or melt driven by the vapor pressure and the recoil of the light pressure. Therefore, the heat- affected zone due to heat diffusion is reduced by the fact that a large amount of the absorbed energy is carried away by the accompanying vaporization.

In summary, a laser micromachining setup for the formation of high-aspect-ratio trenches at oblique angles is developed. The key element of the setup is the insertion of a laser turning mirror between the focusing optics and the plane of the sample. The process has been successfully demonstrated on sapphire wafers; it can therefore be adopted for the dicing of GaN LED dies with angled chip profiles, a geometry which is beneficial for light extraction. Experiments show that there is little compromise on ablation efficiency. Laserinduced damaged, in the form of melt resolidification, is readily removed with a chemical and mechanical treatment.

\section{ACKNOWLEDGMENTS}

This work was jointly supported by a CERG grant of the Research Grant Council of Hong Kong (Project No. HKU 7121/06E) and the University Development Fund of the University of Hong Kong (Nanotechnology Research Institute, Project No. 00600009).

${ }^{1}$ A. Elgawadi, J. Krasinski, G. Gainer, A. Usikov, and V. Dmitriev, J. Appl. Phys. 103, 123512 (2008).

${ }^{2}$ Y. G. Tian and Y. C. Shin, J. Am. Ceram. Soc. 89, 3397 (2006).

${ }^{3}$ Li Lü, J. Fuh, Y.-S. Wong, Laser-Induced Materials and Processes for Rapid Prototyping (Kluwer Academic Publishers, Norwell, 2001).

${ }^{4}$ E. Gu, C. W. Jeon, H. W. Choi, G. Rice, M. D. Dawson, E. K. Illy, and M. R. H. Knowles, Thin Solid Films 453-454, 462 (2004).

${ }^{5}$ T. Otani, L. Herbst, M. Heglin, S. V. Govorkov, and A. O. Wiessner, Appl. Phys. A: Mater. Sci. Process. 79, 1335 (2004).

${ }^{6}$ X. Liu, D. Du, and G. Mourou, IEEE J. Quantum Electron. 33, 1706 (1997).

${ }^{7}$ R. Singh, M. J. Alberts, and S. N. Melkote, Int. J. Mach. Tools Manuf. 48, 994 (2008).

${ }^{8}$ M. R. Krames et al., Appl. Phys. Lett. 75, 2365 (1999).

${ }^{9}$ M. M. Ragheb, H. H. El-Refaei, D. Khalil, and O. A. Omar, J. Lightwave Technol. 25, 2531 (2007).

${ }^{10}$ S. Janz et al., IEEE J. Sel. Top. Quantum Electron. 12, 1402 (2006).

${ }^{11}$ J. M. Kohler and M. Zieren, Thermochim. Acta 310, 25 (1998).

${ }^{12}$ Y. Torisawa, H. Shiku, T. Yasukawa, M. Nishizawa, and T. Matsue, Biomaterials 26, 2165 (2005). 